\title{
Application of Multisensor Information Fusion Technology in the Measurement of Dynamic Machining Errors of Computer Numerical Control (CNC) Machine Tools
}

\author{
Xiaoping $\mathrm{Li} \mathbb{D}^{1,2}$ Yonghong Deng, ${ }^{3}$ and Xuezhe $\mathrm{Li}^{1,2}$ \\ ${ }^{1}$ College of Mechanical and Electrical Engineering, North China Institute of Science and Technology, Langfang 065201, China \\ ${ }^{2}$ Hebei Key Laboratory of Safety Monitoring of Mining Equipment, Langfang 065201, China \\ ${ }^{3}$ School of Electronic and Information Engineering, North China Institute of Science and Technology, Langfang 065201, China
}

Correspondence should be addressed to Xiaoping Li; lixiaoping@ncist.edu.cn

Received 29 September 2021; Accepted 15 October 2021; Published 29 October 2021

Academic Editor: Guolong Shi

Copyright (c) 2021 Xiaoping Li et al. This is an open access article distributed under the Creative Commons Attribution License, which permits unrestricted use, distribution, and reproduction in any medium, provided the original work is properly cited.

\begin{abstract}
A CNC machine tool is process control equipment integrating machine, electricity, and liquid, which makes its fault diagnosis complex and special due to its own advanced, complex, and intelligent characteristics. Traditional diagnostic methods rely on the engineering experience of technical personnel, which incorporates human subjective factors, and can only perform qualitative analysis, resulting in low diagnostic efficiency. And through a single sensor to detect and diagnose the machine tool, the accuracy and credibility of the decision are low, and the system is also weak against interference. In this paper, we first summarize the composition and working principle of $\mathrm{CNC}$ machine tools and analyze the working condition signals generated by $\mathrm{CNC}$ machine tools and the sensors that collect the signals and decide to use a multisensor multisignal fusion-based approach to monitor the machine tool status. It is possible to obtain more effective and valuable information from the observed information through multiple sensors so that the goal of fusion can be achieved. In this paper, a multisensor fusion technique based on wavelet transform and neural network fusion is applied to a machine tool condition monitoring system. The theoretical basis of wavelet analysis and neural network is introduced, and the composition of the condition monitoring system and the process of applying multisensor fusion technology based on wavelet analysis and neural network in the condition monitoring system are given. A complete software and hardware system for online monitoring of CNC machine tools is established. In order to improve the accuracy of the mathematical model, the use of a neural network to fit the nonlinear data and the use of coarse set theory to simplify the relevant data can effectively solve the accurate establishment of the mathematical model in the error compensation method. The thermal error compensation method for CNC machine tools is proposed based on rough set theory, ant colony algorithm, and neural network. This paper first investigates the current development of error compensation technology for CNC machining centers, analyzes the various error sources of CNC machine tools, and finds out the influencing factors affecting the errors of $\mathrm{CNC}$ machine tools.
\end{abstract}

\section{Introduction}

With the development of science and technology and the explosive growth of information, all walks of life, as the foundation of the manufacturing industry, are also flourishing [1]. Nowadays, the production model has been transformed from a single model to an industrialized and information-integrated model and has come to the era of coinciding with the information-physical system, so virtualized industrial resources and the integration of information into the production process have become a popular topic for intelligent manufacturing nowadays [2]. In the transition of production methods, flexible manufacturing systems, computer-integrated manufacturing systems, and networked manufacturing and intelligent manufacturing systems have been proposed one after another [3]. At this time, it is very important to improve the autonomy and synergy of the production system, so various artificial intelligence technologies have been introduced into the design of manufacturing systems. In essence, smart manufacturing is still the concept 
of intelligent devices that use various information generated during the production process to enable analysis, reasoning, judgment, and decision-making [4]. In the previous research on equipment monitoring, only a single sensor was often discussed. In recent years, it has become increasingly common to use multiple sensors of the same type, or multiple sensors of different types, in intelligent systems, and the information collected by the system has become more diverse. However, the effective integration of fused systems composed of multiple sensors into a single system has not been studied enough [5]. Although a lot of research has been done in the past decades to process information from sensor collection systems, the demand for information and the speed of demand are often so great that simply increasing the number of sensors is a solution to meet the system requirements, but this approach results in wasted information and increases the cost of developing intelligent systems [6].

Therefore, how to make full use of the information they provide is an important research element of multisensor systems [7]. The fusion system of information after multisensor information will have a great advantage compared with the system processed by only a single sensor, and the former will not only reflect the real-time status of the measured object more accurately but also collect the status information of the measured object more comprehensively [8]. At this stage, the systematic research on multisensor information fusion technology is not very well developed. Most of the studies are based on the practical problems encountered. The specific implementation plan of the fusion system is determined according to the actual situation and characteristics of the studied target, and the systematic, modular, and standardized formulation is relatively lacking. In addition to the above, the accuracy of the sensors, the speed of computer computing, and human factors and other practical reasons lead to a lack of comprehensive research on such technologies, and in the past, when studying multisensor information fusion systems, less consideration was given to modularity, a high degree of integration, versatility, and applicability in all fields [9]. Multisensor information fusion technology has a broad potential for promotion and application in various fields and has been successfully applied in areas such as military, aerospace, industrial control, and remote sensing. However, large-scale, systematic applications have just sprouted, and the help to production is not large enough. In view of the above, it is necessary to conduct indepth research on the technology of the multisensor information fusion system. Different from traditional machine tools, CNC machine tools are advanced, complex and intelligent, so that its fault diagnosis is complex and special [10]. Causes of CNC machine tool failure are multifaceted, may be mechanical problems, and may also be electrical problems, perhaps both aspects are possible, and the diagnosis is very complex, requiring maintenance personnel in accordance with the actual situation to make a comprehensive judgment. Experience shows that in the CNC machine tool troubleshooting, the time spent on the causes and parts of the fault diagnosis accounted for $80 \%-90 \%$ of the total time, for troubleshooting and repair time accounted for only $10 \%-20 \%$ of the total time [11].
Over the years, for the error compensation method domestically and internationally, the majority of scholars have carried out a lot of research work and also obtained a certain degree of success. But after all, the CNC machine tool machining process is complex, and the cutting process suffered from the impact of tool wear, force, cutting heat, and other reasons. Therefore, there are many reasons that affect machine tool errors. Various sources of error should be fully considered, and an accurate mathematical compensation model should be established to achieve the accuracy of machine tool error compensation. In the CNC machine tool cutting site, the use of inexpensive methods was to detect and identify all the error sources, the use of equipment was to identify and detect the error sources, and the data obtained into the established mathematical model to use the software were to obtain the error compensation value to achieve the purpose of comprehensive error value compensation. The purpose of this thesis research is to build the mathematical model of the thermal error compensation system of CNC machine tools, to realize the compensation correction of the thermal error of $\mathrm{CNC}$ machine tools through software compensation technology, so as to improve the accuracy of CNC machine tool processing through software technology, and to accomplish the achievement of processing a high precision product on a machine tool with low accuracy level and to realize the virtuous cycle of machining accuracy.

\section{Related Work}

High speed, high precision, fast response, and low cost are still the main development directions of CNC machine tools in the future. In ultra-high-precision machining, a high-speed, fast-response, high-resolution, and long-stroke precision positioning control system becomes particularly important, because the high-speed feedback system has the ability to reduce the necessary nonmachining time; thus, the high-speed feedback system has become a direction of development on CNC machine tools [12]. However, it is very difficult to have a high-precision feedback system due to many factors such as clearance and friction between the ball screw and the nut. As the machine tool thermal deformation caused by the thermal error is the main source of error in the machine tool, many researchers' research efforts are focused on the overall thermal error compensation of the machine tool; it is due to the irregular changes in temperature on the machine structure, so that the thermal deformation of time is not a linear process. It expands where it is in contact with the heat source, and the thermal expansion coefficient and the structure of the machine tool system produce complex thermal behavior. Therefore, the selection of the key points affecting the source of thermal error becomes very difficult, and this research content is also very little [13]. In foreign countries, institutions and universities that currently study better thermal error compensation for CNC machine tools include the National Institute of Standards and Technology, the University of Michigan, the University of Cincinnati, the University of Tokyo, the University of Ahern, and the Berlin Institute of Technology. 
Among them, in 1996, the University of Michigan Research Center and SMS jointly developed a comprehensive error compensation supply system, and the development of this system is a collection of thermal error compensation technology, geometric error compensation technology, and load error compensation technology. It has been successfully applied in the company's dual-spindle CNC machine tools. In 1997, the University of Michigan in the United States successfully doubled the machining accuracy of CNC machine tools, with the thermal error compensation technology as the method used implemented in the U.S. General Motors (GM) company's CNC machine tool machining center, so that the manufacturing clutch of this machining center with machining accuracy of more than 100 machine tools has been greatly improved and at the same time also produced huge economic benefits [14]. According to the inspiration triggered by the cerebellar model, in the University of Michigan in the workforce, the machining accuracy of CNC machine tools was successfully improved, the method is based on the structure of the connection controller (CMAC) neural network, and a mathematical model of the machine tool thermal error compensation was artificially constructed [15]. The error online compensation technology was successfully applied by researchers at the University of Michigan to the CNC machining equipment located in the U.S. Boeing aircraft manufacturing company, and the equipment processing accuracy has been significantly improved, such as the huge gantry machining center and its $X$-axis, $Y$-axis and $Z$-axis travel distance of 45 feet, 10 feet, and 4 feet, respectively, through the implementation of online compensation technology; error values were artificially created for the implementation of online compensation technology, which creates artificial error values to balance the original geometric and thermal errors of the machining center, and the machining center's manufacturing accuracy was improved by a factor of 10 , yielding significant economic benefits and pointing the way forward [16]. The U.S. National Institute of Standards and Technology (NIST) is one of the first institutions to study the error compensation technology for CNC machining centers; it compensates for geometric and thermal errors to the machining center area and successfully improves the machining accuracy of the machining center. In recent years, the U.S. National Institute of Standards and Technology (NIST) has been engaged in the study of error control technology, the artificial introduction of new errors is to correct the system error value, that is, through the only measurement of the workpiece in a period of time, and the use of data processing technology is to establish an error compensation model to accurately adjust the original error value of the system [17].

In recent years, Japan in East Asia has also made good achievements in error compensation technology. Based on the new concept of smart manufacturing, researchers at the University of Tokyo have successfully implemented comprehensive error compensation on CNC machine tools using heat as a driving force. Based on the concept of "thermal stiffness," Japanese researchers have proposed the development of machine tool thermal deformation toward CAD and CAM and have achieved good results. Based on fuzzy control theory, a thermal error compensation controller was successfully applied to the TDC-FUZZY spindle head of Osaka Kogyo (OKK) in Japan, and the thermal error of the spindle head was successfully controlled. With the help of the finite element method, researchers at the Technical University of Berlin have calculated the temperature field of the machine components and the whole machine [18].

Neural networks can disperse the learned regular information into the various weights and thresholds of the network, and each node can handle the function of information at the same time. Although the individual neurons are extremely structured and limited in function, numerous neurons achieve extremely colorful behaviors and are widely used. Artificial neural networks have good prospects for development for the following reasons: first, neural networks avoid the shortcomings of expert systems, that is, the need to previously establish a knowledge system, while neural networks obtain knowledge by learning samples to get the weights and thresholds that contain the laws and only need to store the weights and thresholds to achieve the simulation of input and output relationships and to achieve the prediction function, that is, the nonlinear mapping function; second, the neural network has good online learning and parallel operation function, good fault tolerance for parameters, and good inference, especially for nonlinear information, imperfect information, and inaccurate information and has very good simulation ability, as a new modeling technology is widely used [19]. The establishment of CNC machine tools with accurate mathematical models is the focus of error compensation, but with a complex structure of CNC machine tools, access to accurate mathematical models is very difficult, plus the environment of the $\mathrm{CNC}$ machining center is always changing, resulting in machining errors constantly changing, so the compensation value of the error is not a simple addition or subtraction of individual components. It is very difficult to compensate through the linear model of the CNC machining center, and the compensation value is also very inaccurate. But for nonlinear information, the neural network has a good fitting ability and predictive ability. So the neural network can be used as CNC machine tool center error compensation. The input is multiple sets of factors that affect the processing accuracy and the output is the error value corresponding to the corresponding moment factor, and then, the sample data is used for network training [20]. The learned knowledge is stored in the weights and thresholds, and the successful neural network can be learned. The network output value is the error value; that is, the machine will run a certain distance to get the thermal deformation error value. Then, you take the opposite number of the error value and write it into the machine tool to recognize and superimpose on the $\mathrm{CNC}$ center. The $\mathrm{CNC}$ machining center made corresponding actions and successfully realized error compensation [21].

\section{Multisensor-Based Error Analysis and Identification of CNC Machine Tools}

3.1. Sources of Error in CNC Machining. A servo system, a computer numerical control device, input and output 
devices, control media, and the main body of the $\mathrm{CNC}$ machining center constitute a CNC machining center. $\mathrm{CNC}$ machining center processing information is stored in the control medium, commonly known as information carriers. The CNC machine tool to achieve automatic processing of the core is the CNC device, mainly by the communication interface board, position control, PLC interface board, professional function module, computer system board, corresponding control software, and so on. To control system processing input and output information, real-time control of the tool trajectory, for example, is based on the information stored on the control medium. The servo system is the machine tool body, and the CNC system between the links, reverse transmission device, drive control system, servo motor, and position detection system comprise the servo system, that is, the electric drive link. The drive control system commands the servo motor; that is to say, the servo motor is the executive part of the control system.

The comparison of the position command and the actual position signal issued by the computer control system yields the displacement command, which is then amplified by power to guide the machine tool to do very accurate positioning according to the predetermined path and moving speed and finally drive the CNC machine tool to manufacture the workpiece in accordance with the drawing requirements. The detection feedback system is composed of a detection circuit and execution components, obtaining the speed and position information of the moving parts is the purpose of the detection feedback system, and the information obtained is transmitted to the CNC device; the feedback information and the instruction information are compared and corrected with each other, forming a complete closedloop control system. The feed mechanism and spindle, bed, and other mechanical components constitute the main body of the machine tool, which is the mechanical mechanism of the physical part of the CNC machine tool, and the degree of automation of the CNC machine tool is very high. Unlike ordinary machine tools, its productivity and machining accuracy are relatively high, and it also has good vibration resistance. When the machine tool is working, because the gap between the moving parts is small, the amount of wear between each other will be smaller, and it has a good speed change and transmission system and a high degree of automation. The principle of CNC machine tool processing is as follows: the CNC machine tool is a kind of machine tool that uses digital information to achieve automatic control and is compared with ordinary machine tools, and the automation control is the most fundamental difference. When processing mechanical parts on CNC machine tools, as CNC machine tools can only recognize digital information, first of all, the geometric information and process information of the processed workpiece should be turned into digital quantities. Then, according to the requirements of the drawings, we set reasonable tool parameters, process parameters, and technology and then compiled all the processing information of the part into the CNC machine tool to identify the code. Finally, the workpiece processing program is written into the CNC system. After analysis and processing by the computer control system, the control instruction infor- mation is issued, and the CNC machine tool realizes the automatic processing of the workpiece.

The main shaft, various linear guides or rotary axes, columns, and bed constitute the frame structure of a general $\mathrm{CNC}$ machine tool, and the composition of various components may cause various errors. The machining errors of CNC machine tools are composed of the following aspects: (1) interpolation errors generated by the processing of CNC interpolation algorithms; (2) various kinds of errors caused by the cutting force of the machine tool, the gravity of the machine tool, the deformation of the machine tool and its insufficient rigidity, etc.; (3) servo following error caused by the machine tool axis servo system; (4) numerous thermal errors caused by the friction of the internal parts of the machine and the heat exchange of the external environment; (5) assembly errors caused by geometric errors during the assembly process; (6) part size error, that is, the machining accuracy of machine tool parts; and (7) other errors, such as detection errors generated during the measurement process and environmental errors caused by changes in external airflow, air humidity, vibration, etc. The degree of precision of machine tool processing has a significant impact on the overall error which can be divided into two categories of quasistatic error and dynamic error. Quasistatic error is caused by a number of cycles in a certain period of time that basically remain unchanged or change more slowly, and the machine tool itself is closely related to the manufacturing and processing precision of the error, including the CNC machining center caused by the weight of the error, relative movement caused by the error, and thermal expansion and contraction caused by the error and geometric error. The degree of precision of the machine tool and the various sources of error and their influence between the factors are affected as shown in Figure 2. Dynamic error characteristics are more complicated and are generally caused by the following reasons: spindle motion error, machine tool vibration caused by error, servo control system error, etc. Machine tools generally require improvement and promotion of such dynamic error self-processing equipment and self-system.

3.2. CNC Machine Tool Error Identification. Geometric errors mainly come from the machine tool itself, such as the static and dynamic displacement of machine parts, the fit error between machine parts, and manufacturing and processing defects. This is mainly because the pitch of the ball screw changes with the positioning error, angular movement error, and straightness error caused by the deformation of the guide rail. The servo system is mismatched due to the backlash error caused by the backlash of the transmission gear, ball screw, etc. Geometric error is only related to the relative position of the cutting tool or the workpiece, as shown in Figure 3. Take the CMM as an example, and it generally includes linear motion axes $(X, Y$, and $Z)$, which are perpendicular to each other. In general, the position of the machined workpiece and the probe with respect to each other is accomplished by the relative motion of the working parts with respect to each other. However, in actual applications, due to factors such as part lubrication, installation, 


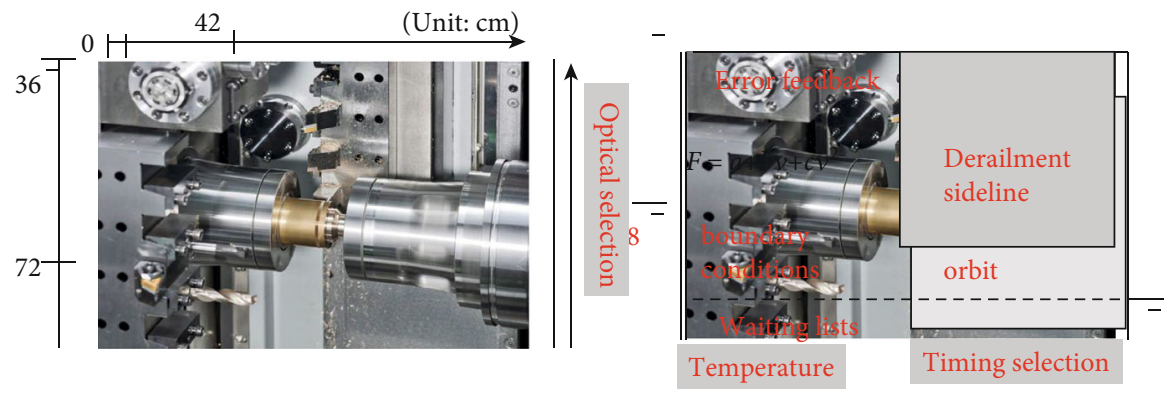

FIGURE 1: Error analysis of CNC machining.

manufacturing errors, servo system following errors, and clearance errors, many working parts of the machine tool are performing the motion detection required by the program, and the corresponding motion errors will occur. The geometric error of the CNC machining center is a comprehensive mapping of each important part of the machine tool after the installation of the comprehensive geometric error. According to the kinematic theory, each workpiece in space is composed of six degrees of freedom with three rotational degrees of freedom and three translational degrees of freedom. Ideally, however, only one degree of freedom of relative motion is carried out in each zero part of the machine, and the other five degrees of freedom of motion in the other directions are limited by the guideways. But in fact, when the work moves in one direction, it is accompanied by the error movement in the other five directions.. It is because of the many factors affecting the geometric error of CNC machine tools, so it is difficult to accurately detect, but because the geometric error has the characteristics of stability, this paper establishes the compensation model, the geometric error data added to the establishment of the model, the use of the machine tool itself in the real-time online measurement system, and the real-time measurement feedback to the CNC center; the CNC center will be these data into the neural network for prediction compensation.

In the drive system of high-precision and high-speed CNC machine tools, thermal error caused by thermal expansion and contraction is the main error source of $\mathrm{CNC}$ machining centers, so the research object of this paper is thermal error. The thermal error is caused by the thermal expansion and contraction of $\mathrm{CNC}$ machining center components, and the thermal deformation is caused by the complex temperature field when the machine tool is working, so the complex temperature field is fundamental to the study of thermal error and is the main research content of this paper. The temperature field of the machine tool is mainly the system being affected by internal and external heat sources; thus, the change in temperature to the unstable stage of thermal equilibrium in the machine tool components causes a decline in the degree of the processing precision and heat transfer deformation process. In machine tools, there are two forms of thermal errors.

(1) Thermal errors and location are related to the uneven heat generated by the machine tool, which will cause thermal deformation of the guide rail, thus causing angular motion errors and straightness errors that follow the changes in the temperature field of the machine tool. For example, due to the increase in the measurement scale, the temperature field of the machine tool changes, and the change of the pitch error is reduced, followed by the positioning error of the following temperature field. The thermal deformation of the machine tool will also cause the verticality between the machine coordinate axes to change, resulting in verticality errors

(2) Thermal errors and location are not related, such as the machine tool dragging plate and the machine tool spindle thermal movement. To two coordinate vertical machining centers, for example, the general heat source is divided into $X-, Y$-, and $Z$-axis track vice friction, box gear friction of the spindle, $X-, Y$-, and $Z$-axis ball screw nut friction, $X, Y$, and $Z$ drive motor, oil pump, spindle bearing friction, and the spindle motor. At the same time, the support parts of the CNC machining center can also be the main form of the heat source causing thermal deformation, among which the tool changer drive motor, electrical parts, and power supply are the most common. If the machining center is being processed, the cooling pump is on, with the cutting heat of the coolant, and will become one of the main sources of heat deformation caused by the machine tool

3.3. Multisensor-Based Error Measurement. With the development of science and technology and the progress of basic industries, the market for high-precision products is becoming more and more extensive. Whether it is the electronics industry or the aerospace industry, precision machining technology has been widely used in all walks of life. Whether it is a highly integrated microprocessor chip or a huge international space station, it is inseparable from high-reliability, high-precision CNC machine tools. Now, there are mainly two methods of error compensation and error prevention to reduce the various errors of CNC machine tools. This paper mainly focuses on the study of thermal errors, so the next focus is on reducing thermal errors using error compensation methods and error prevention methods. Numerical control machine tools will be affected by various heat sources during the working process. The work of the CNC machining center is always under the alternating action of 


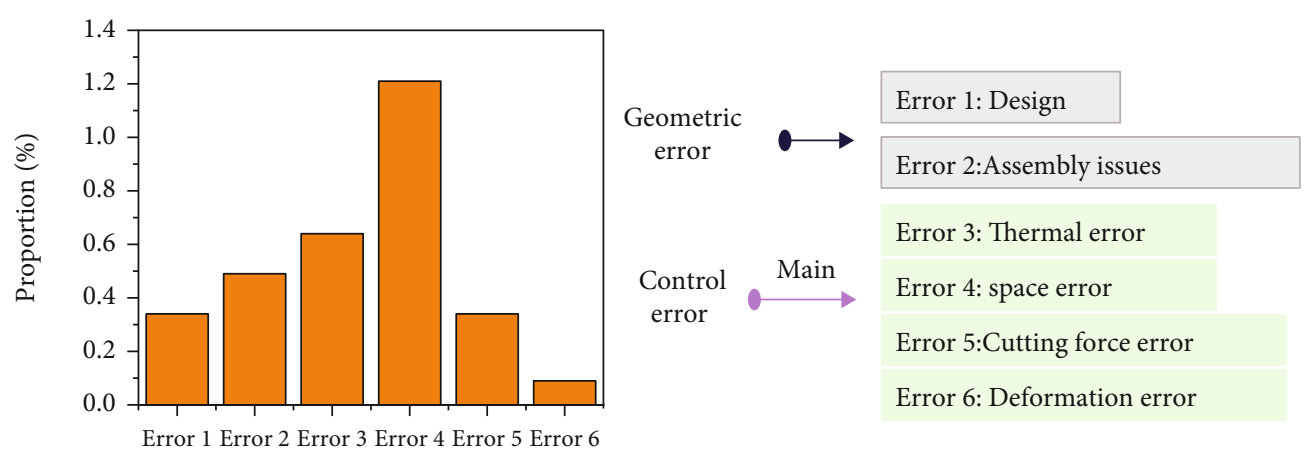

FIGURE 2: Main error sources of the machine.

external and internal heat sources, and these heat sources have nonconstant characteristics. And with the different processing conditions, the degree of heat caused is different. With different structures, shapes, and materials, CNC machining centers have different thermal inertias, different thermal resistances between connections, and different heat transfer conditions. Thus, the formation of different temperature fields is on the surface of the machine tool. The formation of the temperature field is caused by the thermal expansion and contraction of machine tool components, the location of the machine tool components has changed, the machine tool's previous processing accuracy was destroyed, and different temperature fields further accelerate the wear and tear of the moving parts, which caused the error of the machined workpiece. By the CNC machine tool thermal error factors and its nature of performance, error compensation technology and error prevention technology are the main measures to reduce the machine tool thermal error. The error prevention method is to reduce the CNC machine tool thermal error of hard technology, generally by modifying the machine structure and controlling the heat source, and there are other methods to achieve control of the machine tool thermal error function. Commonly used methods are as follows.

(1) Reasonably optimize the layout of the machine structure, that is, to optimize the machine structure and layout design. In the optimization of the structure, one should try to make the machine tool components uniformly heated, so that the relative position remains unchanged; the specific measure is for the machine tool to generate heat for uniform conduction; this method can effectively reduce the amount of relative heat deformation of the machine tool. In addition, in the design of machine tools, symmetrical structure can effectively reduce the thermal deformation brought about by thermal errors to the gantry surface grinding machine; for example, if only designed as a single-column structure, having asymmetric results, it is easy to trigger deformation bending, but if you use a symmetrical structure double-column type, there is only vertical movement

(2) Equalize the machine temperature field and set up an auxiliary heat source
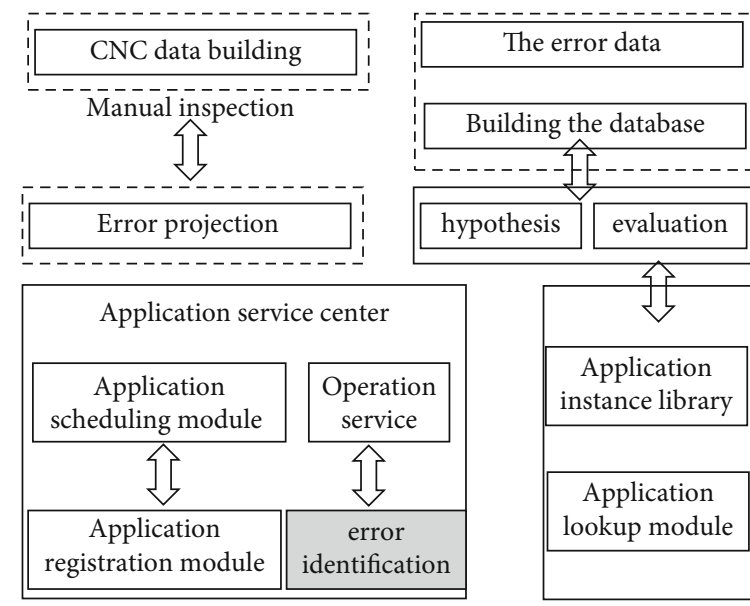

Figure 3: CNC machine error identification.

(3) Reduce the internal heat generation and heat sources of the machine tool. Reducing thermal errors can be achieved by controlling the internal heat-generating device of the machine tool, thus alleviating the heat transformation and frictional heat generation of the machining center and reducing the transmission gear and drive shaft

(4) Improve the heat dissipation and insulation conditions, i.e., to improve the cooling and lubrication during the machining process. In the process of the workpiece being machined, a lot of heat is generated, which needs to be cooled, and at the same time, chip removal should be done quickly; a hydraulic pump station is the equipment that generates more heat in the hydraulic system, so it should be placed outside the machine tool, but if it cannot be placed outside, effective heat transfer measures should be taken; in order to improve the heat transfer capability, forced lubrication cooling is used in the CNC machining center spindle parts or spindle box, sometimes circulating the cooling oil after cooling for cooling; for the place of high heat generation, it should increase its heat dissipation area

(5) Use the constant temperature environment, through the effective management of machine tool processing environment temperature, to reduce the purpose of 
thermal error. Generally, it should be used in a constant temperature workshop with high-precision $\mathrm{CNC}$ machine tools, and preheating is done, so that the temperature of the components rises evenly to reduce the amount of thermal deformation and the machine tool processing accuracy can be effectively improved

In the study of CNC machine tool thermal errors very early, error prevention methods have attracted the attention of scholars and obtained good results, for reducing the thermal error of the machine tool has played an important role. However, along with the progress of science and technology and research workers, the shortcomings of the error prevention method have been fully exposed. First of all, in terms of improving the machining accuracy of machine tools, only error-proofing methods can be adopted. When the accuracy reaches a certain level, it will become very difficult to improve the machining accuracy of CNC machine tools, especially for ultraprecision machining processes. Secondly, the use of this method to process products with high-precision requirements will significantly increase the cost, which is sometimes greater than the economic benefits that can be brought by reducing processing errors. There are two main reasons for the limitations of the use of error prevention methods: first, in the process of machine tool processing, the external environment and processing conditions are not constant and the environment has been changing and is unpredictable, causing the machine tool's own errors, and then, the machine tool processing itself has errors and the two errors are not constant, so to prevent or accurately estimate these errors in advance is often not easy. Second, with the complexity of the machine tool, the machine tool is composed of numerous electronic and mechanical components; any one of them generated by the error may increase the overall error of the machine tool. And strictly limiting the error of each component to control the source of error is generally hard to do, and even if you can do it, it is not an economic method. The error compensation method and error prevention method are different; through the analysis of different sources of error on the impact of machining accuracy, an error compensation mathematical model was constructed, so as to artificially modify the distance of the machine tool, so that the machine tool machining accuracy can be significantly improved. Compared with the error prevention as a hard technique, the error compensation method is a soft technique. This method does not need to modify the machine tool hardware, and you can use the general degree of precision machine tools to produce high-precision products, to achieve the purpose of producing high-precision parts through low-precision equipment.

\section{Error Compensation for Dynamic Processing Based on Multiple Sensors}

For the thermal expansion and contraction of CNC machine tools, there are two compensation methods: first, when high precision is required, the online compensation system is used to compensate for the error value; this method requires first establishing a mathematical model of temperature and thermal error law, using a real-time measurement system to obtain the current state of the machine tool, and then inputting them into the error compensation mathematical model to achieve real-time compensation of thermal error; second, under lower-precision requirements, you can use the method of preset compensation, through offline modeling or direct detection of the thermal deformation values, to obtain the mathematical model of thermal deformation and influencing factors and the resulting deformation law for programming and finally to achieve thermal error compensation to improve the machining accuracy of $\mathrm{CNC}$ machining centers, as shown in Figure 4.

CNC machine tool error compensation was earlier explored in the process offline to gain experience in changing the CNC code to make error compensation possible. The compensation process is time-consuming and also requires the assumption that the error obtained offline and the actual error value must be the same. Over time, two different error compensation techniques were formed: the feedback interruption method and the origin translation method. There are many reasons for thermal deformation of CNC machine tools. In the working process of CNC machine tools, it will be affected by various heat sources, including mechanical source power components, such as feed motors and spindle motors. They consume energy and convert it into heat, that is, frictional heat caused by transmission components (such as vises and bearing vises). The tool cutting the workpiece will cause cutting heat. Environmental heat is caused by temperature changes in the processing workshop and sunlight in the workshop, as shown in Figure 5. In the internal and external heat sources under the influence of the machine tool, always in a nonconstant heat source under the work, along with the different cutting and processing conditions, the degree of impact of the heat source is not the same. Machine tools form different temperature fields, because of the structure, shape, and material of machine tool components, and the thermal inertia of various machine tools are different. The thermal resistances of the bonding surfaces between the connecting devices form different temperature fields. Different temperature fields are caused by the relative position change between the machine tool parts, thermal expansion, and cold contraction, thereby reducing the original accuracy of the machine tool, further accelerating the wear of the machine tool parts, and finally causing thermal errors in the machine tool processing workpieces.

Compared with CMM, the CNC cutting machining center has a complex measurement environment, and there are many factors affecting the error, so it is difficult to get very accurate measurement values, as shown in Figure 6. In addition, the thermal error is caused by many factors, and the thermal error has a nonlinear and mutual influence; all the conditions need to be simulated to identify and detect the thermal error, which generally takes a very long time but also cannot be done directly in the computer for a variety of errors in real-time compensation and correction of measurement data. Based on the above reasons, we propose to optimize the grouping of $\mathrm{CNC}$ machine tool thermal errors to establish a mathematical model. In the machine tool thermal 


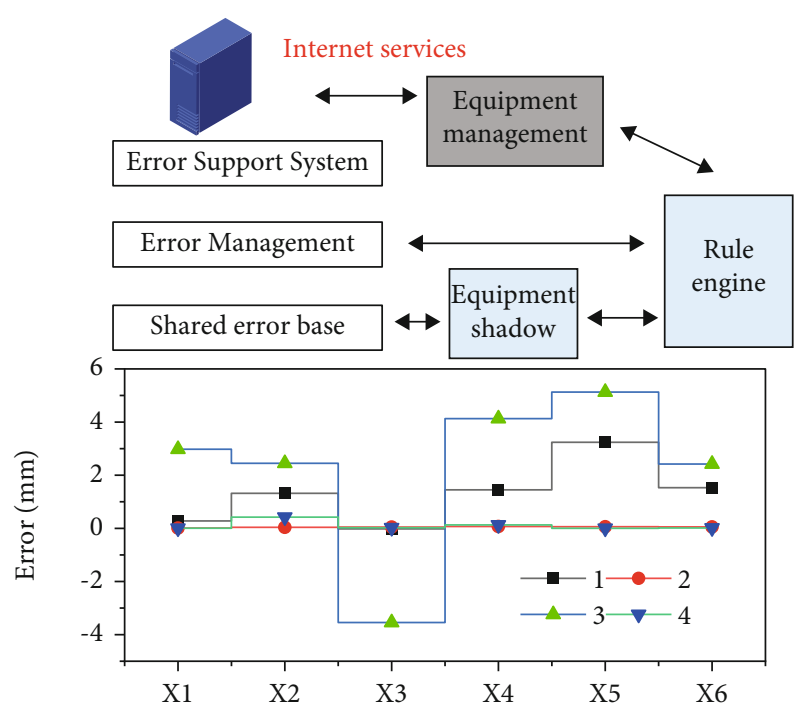

FIGURE 4: Error compensation for dynamic machining.

error to establish a mathematical model, the first need by the interrelationship between temperature variables, the measured temperature variables are grouped, followed by the knowledge of the rough set theory. In order to identify the relationship between the temperature variable and the thermal error, the important parameter characteristics that affect the thermal error of the machine tool are selected. Combining typical temperature variables to simplify the machine tool temperature error compensation system, we grouped and established an artificial neural network machine tool error compensation system to avoid the coupling between temperature variables in the error model. This further improves the robustness and accuracy of the CNC machine tool thermal error compensation model, and finally effectively compensates the real-time thermal error of the CNC machine tool. At the same time, the ant colony algorithm is used in the network training process to make the network quickly reach the global optimal value, thereby improving the prediction accuracy of the model. Finally, the weights and thresholds obtained from the network training are input to the machine tool according to a certain algorithm to achieve the correction of the machine tool position.

The data was measured in a machine tool factory. By analyzing the structure of the GMC2000A machine tool, it was determined that the heat sources at key points of the machine tool were the motor housing, motor plate, cross beam, scale, slider, nut holder, cross slide left, cross slide right, environment, and nine other locations. The test method is performed as follows: nine temperature sensors are arranged on the GMC2000A machine to obtain the temperature values of nine key heat sources, the machine runs at $6-12 \mathrm{~m} / \mathrm{min}$, and the position error values of the $Y$-axis of the GMC2000A machine are collected using a paperless recorder and a laser interferometer, and the positioning precision is measured using a program that stops for 5 seconds every $170 \mathrm{~mm}$ of travel, with a total travel of $3400 \mathrm{~mm}$. A total of 840 error data points were collected during the two days. 40 sets of temperature data were also collected during the period (one set of

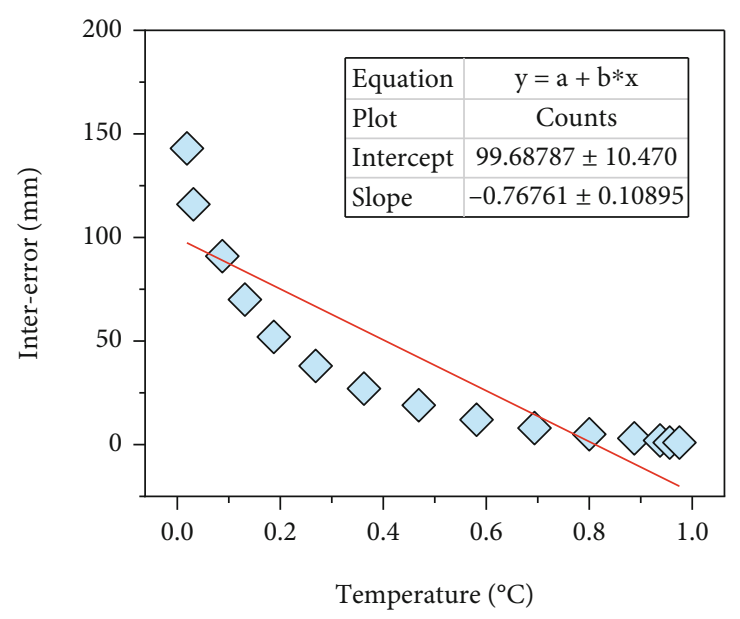

FIGURE 5: Temperature measurement error graph.

temperature values was collected for one stroke because the temperature value did not change much during one stroke of the machine). The maximum temperature rise was 8.06 degrees, and the maximum error value was $111 \mu \mathrm{m}$, as shown in Figure 7.

Generally speaking, in the thermal error compensation control system of CNC machine tools, the condition attribute temperature is the choice of thousands of measuring points. However, in practical applications, if there are too many temperature measuring points, not only will the workload of the measuring points and the temperature measuring points be arranged too close but also the output values of adjacent measuring points will have a great correlation. In order to achieve the purpose of obtaining a suitable number of measurement points, selected from a large number of measurement point temperature, and under the condition of ensuring the same inscription ability or the same differentiation ability, that is, the condition attribute uniquely determining the decision attribute, it is necessary to simplify the measurement point temperature in the $\mathrm{CNC}$ machine tool thermal error compensation system. Therefore, it is known from the knowledge of rough set theory that the optimization of the conditional attribute temperature measurement points can be achieved by optimizing the error-influencing conditional attributes of the machine tool thermal error compensation system, as shown in Figure 8. In the machine tool thermal error to establish a mathematical model, the first need by the interrelationship between temperature variables, the measured temperature variables are grouped, followed by the knowledge of the rough set theory.

\section{Conclusion}

Relying on improving the machining accuracy and assembly accuracy of mechanical parts is costly and difficult, which restricts the further improvement of $\mathrm{CNC}$ machine tool machining accuracy. Along with the generation of big data processing and intelligent computing technology, the artificial creation of an error to offset the original error of the machine tool shows attractive prospects, and through this 


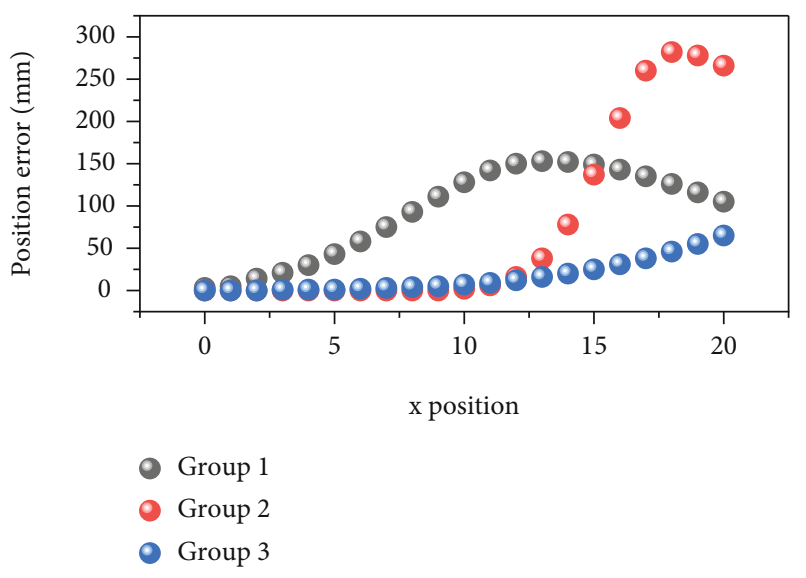

FiguRe 6: Error curve.

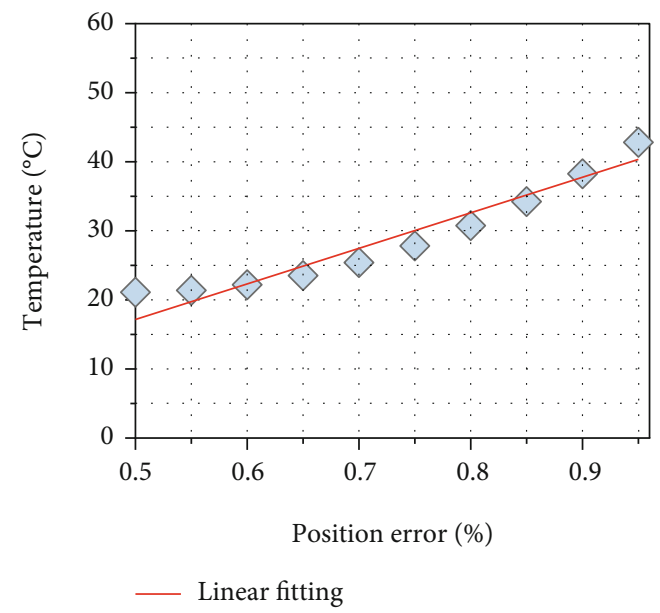

Figure 7: Temperature profile.

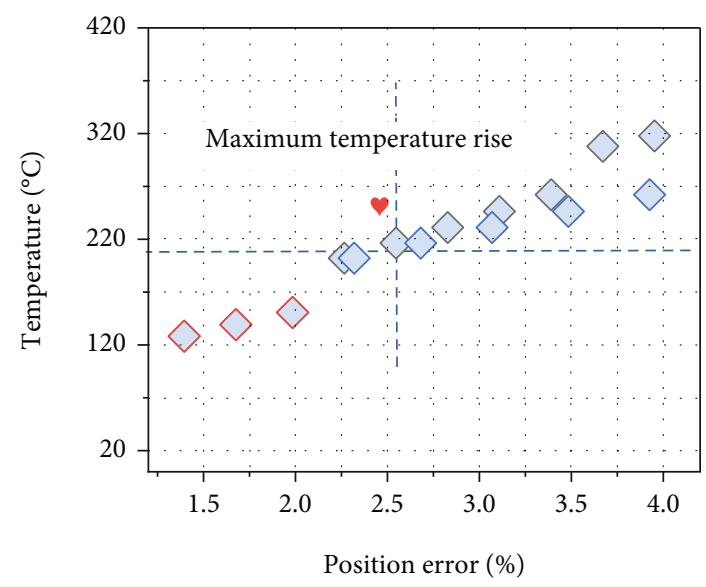

FIgURE 8: Thermal error at key points.

error compensation method to achieve simple, scalable, low cost, will become a powerful tool to improve the machining accuracy of CNC machine tools. This paper first investigates the current development of error compensation technology for $\mathrm{CNC}$ machining centers, analyzes the various error sources of CNC machine tools, and finds out the influencing factors affecting the errors of CNC machine tools. Secondly, it introduces the current methods of intelligent computing, the rough set theory can realize the simplification of data, the BP neural network has the ability to handle uncertain information and arbitrarily fit nonlinear data, has a good learning cognitive function, stores the knowledge mined from the data in different weights and thresholds, and implements simultaneous operation and other advantages, and the ant colony algorithm can realize the global optimization search, so the use of ant colony. Therefore, the weights and thresholds obtained by the ant colony algorithm are given to the network as initial values, which can effectively avoid the BP neural network from falling into local optima during the learning process. The next step is to install the sensor at the key heat source point on the GMC2000A machine tool, collect the data, process the data by using the rough set theory, establish the mathematical model through the BP neural network, train and learn the network by using the characteristics of the global optimization search of the ant colony algorithm in the learning process of the network, and obtain the weights and thresholds that contain the laws between the heat source and the thermal error. In the future, the thermal error compensation method for CNC machine tools is proposed based on rough set theory, ant colony algorithm, and neural network.

\section{Data Availability}

The data used to support the findings of this study are available from the corresponding author upon request.

\section{Conflicts of Interest}

The authors declare that they have no known competing financial interests or personal relationships that could have appeared to influence the work reported in this paper.

\section{Acknowledgments}

This work is supported by the Fundamental Research Funds for the Central Universities (Nos. 3142018049, 3142019055, and 3142017011), School Education and Teaching Reform Funding Project (No. HKXNJG2021049), and Science and Technology Support Program of Hebei Province (No. 17211826).

\section{References}

[1] X. Hao, Y. Li, Z. Zhao, and C. Liu, "Dynamic machining process planning incorporating in-process workpiece deformation data for large-size aircraft structural parts," International journal of computer integrated manufacturing, vol. 32, no. 2, pp. 136-147, 2019.

[2] R. Huang, J. Jiang, B. Huang, and S. Zhang, "Multilevel structured NC machining process model based on dynamic machining feature for process reuse," The international journal of advanced manufacturing technology, vol. 104, no. 5-8, pp. 2045-2060, 2019. 
[3] J.-P. Hung and W.-Z. Lin, "Investigation of the dynamic characteristics and machining stability of a bi-rotary milling tool," Advances in science and technology research journal, vol. 13, no. 1, pp. 14-22, 2019.

[4] Y. Jin, M. Wang, Z. Zuo et al., "Diagnostic value and dynamic variance of serum antibody in coronavirus disease 2019," International journal of infectious diseases, vol. 94, pp. 49-52, 2020.

[5] F. Kahwash, I. Shyha, and A. Maheri, "Dynamic simulation of machining composites using the explicit element-free Galerkin method," Composite structures, vol. 198, pp. 156-173, 2018.

[6] C. Liu, Y. Li, and W. Shen, "A real time machining error compensation method based on dynamic features for cutting force induced elastic deformation in flank milling," Machining Science and Technology, vol. 22, no. 5, pp. 766-786, 2018.

[7] G. Liu, J. Hong, W. Wu, and Y. Sun, "Investigation on the influence of interference fit on the static and dynamic characteristics of spindle system," The International journal of advanced manufacturing technology, vol. 99, no. 5-8, pp. 1953-1966, 2018.

[8] J. Liu, H. Zhou, X. Liu et al., "Dynamic evaluation method of machining process planning based on digital twin," IEEE Access, vol. 7, pp. 19312-19323, 2019.

[9] O. Naparstek and K. Cohen, "Deep multi-user reinforcement learning for distributed dynamic spectrum access," IEEE transactions on wireless communications, vol. 18, no. 1, pp. 310-323, 2019.

[10] A. Rambaut, E. C. Holmes, Á. O'Toole et al., “A dynamic nomenclature proposal for Sars-Cov-2 lineages to assist genomic epidemiology," Nature Microbiology, vol. 5, no. 11, pp. 1403-1407, 2020.

[11] J. Schlemper, J. Caballero, J. V. Hajnal, A. N. Price, and D. Rueckert, "A deep cascade of convolutional neural networks for dynamic MR image reconstruction," IEEE transactions on medical imaging, vol. 37, no. 2, pp. 491-503, 2018.

[12] J. Schoop, H. A. Poonawala, D. Adeniji, and B. Clark, “AIenabled dynamic finish machining optimization for sustained surface integrity," Manufacturing Letters, vol. 29, pp. 42-46, 2021.

[13] W. Takahashi, N. Suzuki, and E. Shamoto, "Development of a novel boring tool with anisotropic dynamic stiffness to avoid chatter vibration in cutting: part 1: design of anisotropic structure to attain infinite dynamic stiffness," Precision engineering journal of the international societies for precision engineering and nanotechnology, vol. 68, pp. 57-71, 2021.

[14] W. Takahashi, N. Suzuki, and E. Shamoto, "Development of a novel boring tool with anisotropic dynamic stiffness to avoid chatter vibration in cutting: part 2: analytical and experimental verification of the proposed method," Precision engineering journal of the international societies for precision engineering and nanotechnology, vol. 68, pp. 20-34, 2021.

[15] M. A. Tamarkin, E. E. Tishchenko, and A. S. Shvedova, "Optimization of dynamic surface plastic deformation in machining," Russian engineering research, vol. 38, no. 9, pp. 726-727, 2018.

[16] L.-J. Wang, L.-M. Chen, Y. Chen et al., "Ultrasonography assessments of optic nerve sheath diameter as a noninvasive and dynamic method of detecting changes in intracranial pressure," Jama Ophthalmology, vol. 136, no. 3, pp. 250-256, 2018.

[17] S. Wang, H. Liu, P. H. Gomes, and B. Krishnamachari, "Deep reinforcement learning for dynamic multichannel access in wireless networks," IEEE transactions on cognitive communications and networking, vol. 4, no. 2, pp. 257-265, 2018.

[18] W. Wei, X. Fan, H. Song, X. Fan, and J. Yang, "Imperfect information dynamic Stackelberg game based resource allocation using hidden Markov for cloud computing," IEEE transactions on services computing, vol. 11, no. 1, pp. 78-89, 2018.

[19] W. Wu, Z. Huang, J. Zeng, and K. Fan, “A fast decisionmaking method for process planning with dynamic machining resources via deep reinforcement learning," Journal of manufacturing systems, vol. 58, pp. 392-411, 2021.

[20] F. Xiang, X. Wang, X. He et al., "Antibody detection and dynamic characteristics in patients with coronavirus disease 2019," Clinical Infectious Diseases, vol. 71, no. 8, pp. 19301934, 2020.

[21] G. Xiao, Y. He, Y. Geng, Y. Yan, and M. Ren, "Molecular dynamics and experimental study on comparison between static and dynamic ploughing lithography of single crystal copper," Applied Surface Science, vol. 463, pp. 96-104, 2019. 